\title{
HIERARCHICAL STAR FORMATION IN NEARBY LEGUS GALAXIES
}

\author{
Debra Meloy Elmegreen ${ }^{1}$, Bruce G. Elmegreen $^{2}$, Angela Adamo $^{3,4}$, Alessandra Aloisi $^{5}$, Jennifer Andrews ${ }^{6}$, \\ Francesca Annibali $^{7},{\text { Stacey N. } \text { Bright }^{5} \text {, Daniela Calzetti }}^{6}$, Michele Cignoni ${ }^{5}$, Aaron S. Evans ${ }^{8,9}$, \\ John S. Gallagher III ${ }^{10}$, Dimitrios A. Gouliermis ${ }^{3,11}$, Eva K. Grebel ${ }^{12}$, Deidre A. Hunter ${ }^{13}$, Kelsey Johnson ${ }^{8}$, \\ Hwihyun Kim ${ }^{14}$, Janice LeE ${ }^{5}$, Elena Sabbi ${ }^{5}$, Linda J. Smith ${ }^{15}$, David Thilker ${ }^{16}$, Monica Tosi ${ }^{7}$, and Leonardo Ubeda ${ }^{5}$ \\ ${ }^{1}$ Vassar College, Department of Physics and Astronomy, Poughkeepsie, NY 12604, USA \\ 2 IBM Research Division, T.J. Watson Research Center, Yorktown Hts., NY 10598, USA \\ ${ }^{3}$ Max Planck Institut für Astronomie, Königstuhl 17, D-69117 Heidelberg, Germany \\ ${ }^{4}$ Department of Astronomy, Oskar Klein Centre, Stockholm University, AlbaNova University Centre, SE-106 91 Stockholm, Sweden \\ ${ }^{5}$ Space Telescope Science Institute, 3700 San Martin Drive, Baltimore, MD 21218, USA \\ ${ }_{7}^{6}$ Department of Astronomy, University of Massachusetts, Amherst, MA 01003, USA \\ ${ }^{7}$ INAF-Osservatorio Astronomico di Bologna, Via Ranzani 1, I-40127 Bologna, Italy \\ ${ }^{8}$ Department of Astronomy, University of Virginia, P.O. Box 400325, Charlottesville, VA 22904-4325, USA \\ ${ }^{9}$ National Radio Astronomy Observatory, 520 Edgemont Road, Charlottesville, VA 22903, USA \\ ${ }^{10}$ Department of Astronomy, University of Wisconsin-Madison, WI 53706, USA \\ ${ }^{11}$ Universität Heidelberg, Zentrum für Astronomie, Institut für Theoretische Astrophysik, Albert-Ueberle-Str. 2, D-69120 Heidelberg, Germany \\ 12 Astronomisches Rechen-Institut, Zentrum für Astronomie der Universität Heidelberg, Mönchhofstr. 12-14, D-69120 Heidelberg, Germany \\ ${ }^{13}$ Lowell Observatory, 1400 West Mars Hill Road, Flagstaff, AZ 86001, USA \\ ${ }^{14}$ School of Earth and Space Exploration, Arizona State University, Tempe, AZ 85287, USA \\ ${ }^{15}$ Space Telescope Science Institute and European Space Agency, Baltimore, MD 21218, USA \\ ${ }^{16}$ Department of Physics and Astronomy, Johns Hopkins University, 3701 San Martin Drive, Baltimore, MD 21218, USA \\ Received 2014 March 31; accepted 2014 April 22; published 2014 May 8
}

\begin{abstract}
Hierarchical structure in ultraviolet images of 12 late-type LEGUS galaxies is studied by determining the numbers and fluxes of nested regions as a function of size from $\sim 1$ to $\sim 200 \mathrm{pc}$, and the number as a function of flux. Two starburst dwarfs, NGC 1705 and NGC 5253, have steeper number-size and flux-size distributions than the others, indicating high fractions of the projected areas filled with star formation. Nine subregions in seven galaxies have similarly steep number-size slopes, even when the whole galaxies have shallower slopes. The results suggest that hierarchically structured star-forming regions several hundred parsecs or larger represent common unit structures. Small galaxies dominated by only a few of these units tend to be starbursts. The self-similarity of young stellar structures down to parsec scales suggests that star clusters form in the densest parts of a turbulent medium that also forms loose stellar groupings on larger scales. The presence of super star clusters in two of our starburst dwarfs would follow from the observed structure if cloud and stellar subregions more readily coalesce when self-gravity in the unit cell contributes more to the total gravitational potential.
\end{abstract}

Key words: galaxies: ISM - galaxies: star clusters: general - ISM: structure - stars: formation

Online-only material: color figures

\section{INTRODUCTION}

Interstellar turbulence produces hierarchical structure in the gas (Kritsuk et al. 2013) and in the stars that form from this gas (see review in Elmegreen 2010), leading to nested young stellar regions with flocculent spiral arms (Elmegreen et al. 2003) and star complexes (Efremov 1995) on kpc scales, OB associations on $100 \mathrm{pc}$ scales (Gouliermis 2011), and dispersed and bound clusters on parsec scales (Feitzinger \& Braunsfurth 1984; Gomez et al. 1993; Larson 1995; Scheepmaker et al. 2009; Bastian et al. 2011). The bound clusters themselves appear to be the densest parts of this hierarchy, where the fraction of the local gas mass that is dense enough to form stars is high, and so the efficiency of star formation in the region is also high (Elmegreen 2008; Parmentier \& Fritze 2009).

Hierarchical structure in young stellar regions is widespread and may be characteristic of all star formation. Still, there is considerable variation in gravitational binding of the clusters that form (Larsen \& Richtler 2000; Maíz-Apellániz 2001). The most massive star-forming regions in the Milky Way are mostly unbound, such as W43, which spans $300 \mathrm{pc}$, containing $7 \times 10^{6} M_{\odot}$ of molecular gas and the potential to form bound clusters up to $\sim 10^{5} M_{\odot}$ (Nguyen Luong et al. 2011). On the other hand, some starburst galaxies (e.g., Whitmore et al. 2010), including dwarf irregular starbursts like NGC 1569 (Hunter et al. 2000) and NGC 1705 (Annibali et al. 2009), have starforming regions with about the same total mass, $10^{6} M_{\odot}$, but concentrated within tightly bound cores spanning only several tens of pc. We would like to understand why some regions form bound clusters and others do not.

The kinematic pressure from stellar motions in a massive cluster is $\sim 10^{8} k_{\mathrm{B}}$, much higher than the average molecular cloud pressure, $10^{6} k_{\mathrm{B}}$ for Boltzmann's constant $k_{\mathrm{B}}$ (Tan et al. 2013). Loose stellar groupings have lower kinematic pressures than clusters. It seems logical that higher pressures produce a higher fraction of star formation in the form of bound clusters (Elmegreen 2008). High pressure correlates with high gas surface density in a galaxy and therefore with high areal star formation rate (Kennicutt 1998), possibly giving the correlation between bound cluster fraction and star formation rate found by Larsen \& Richtler (2000), Goddard et al. (2010) and Adamo et al. (2011). Similarly on smaller scales, the Orion region has a higher pressure than the Sco-Cen region and Orion also has a higher clustering fraction (Elias et al. 2009). The extent to which 
Table 1

Linear Fits to Correlations

\begin{tabular}{|c|c|c|c|c|c|c|c|c|}
\hline Galaxy & Type & $\begin{array}{c}D^{\mathrm{a}} \\
(\mathrm{Mpc})\end{array}$ & $B_{\mathrm{NS}}$ & $B_{\mathrm{NS}, 275}$ & $B_{\mathrm{TS}}$ & $B_{\mathrm{FS}}$ & $B_{\mathrm{NF}}$ & $B_{\mathrm{OS}}$ \\
\hline NGC 1566 & SABbc & 13.20 & $-1.34 \pm 0.05$ & $-1.30 \pm 0.04$ & $-0.33 \pm 0.05$ & $1.00 \pm 0.03$ & $-1.33 \pm 0.05$ & $0.75 \pm 0.12$ \\
\hline NGC 1705 & SA0pec [Irr] & 5.10 & $-1.86 \pm 0.10$ & $-1.89 \pm 0.30$ & $-0.16 \pm 0.01$ & $1.70 \pm 0.10$ & $-1.09 \pm 0.01$ & $0.10 \pm 0.28$ \\
\hline NGC 2500 & SBd & 10.10 & $-1.17 \pm 0.06$ & $-1.18 \pm 0.05$ & $-0.31 \pm 0.07$ & $0.85 \pm 0.03$ & $-1.36 \pm 0.09$ & $0.86 \pm 0.09$ \\
\hline NGC 3738 & $\mathrm{Im}$ & 4.90 & $-1.39 \pm 0.06$ & $-1.39 \pm 0.05$ & $-0.60 \pm 0.05$ & $0.80 \pm 0.02$ & $-1.75 \pm 0.06$ & $0.92 \pm 0.09$ \\
\hline NGC 5253 & Im pec & 3.15 & $-1.51 \pm 0.08$ & $-1.52 \pm 0.14$ & $-0.49 \pm 0.06$ & $1.03 \pm 0.05$ & $-1.47 \pm 0.06$ & $1.00 \pm 0.06$ \\
\hline NGC 5477 & SAm & 6.40 & $-0.98 \pm 0.06$ & $-1.14 \pm 0.06$ & $-0.17 \pm 0.04$ & $0.81 \pm 0.05$ & $-1.21 \pm 0.06$ & $0.36 \pm 0.15$ \\
\hline NGC 7793 & SAd & 3.44 & $-1.62 \pm 0.08$ & $-1.62 \pm 0.09$ & $-0.41 \pm 0.07$ & $1.21 \pm 0.05$ & $-1.34 \pm 0.06$ & $0.42 \pm 0.11$ \\
\hline IC 4247 & $\mathrm{~S} ?[\mathrm{Irr}]$ & 5.11 & $-1.14 \pm 0.04$ & $-1.17 \pm 0.04$ & $-0.40 \pm 0.06$ & $0.75 \pm 0.02$ & $-1.53 \pm 0.09$ & $0.63 \pm 0.06$ \\
\hline IC 559 & Sc [Irr] & 5.30 & $-1.12 \pm 0.14$ & $-1.13 \pm 0.08$ & $-0.39 \pm 0.06$ & $0.74 \pm 0.14$ & $-1.47 \pm 0.13$ & $1.16 \pm 0.30$ \\
\hline ESO486-G021 & $\mathrm{S} ?[\mathrm{Irr}]$ & 9.50 & $-1.47 \pm 0.08$ & $-1.32 \pm 0.09$ & $-0.45 \pm 0.10$ & $1.02 \pm 0.03$ & $-1.43 \pm 0.11$ & $0.72 \pm 0.06$ \\
\hline UGC 695 & $\mathrm{~S} ?[\mathrm{Irr}]$ & 10.90 & $-1.83 \pm 0.15$ & $-1.70 \pm 0.10$ & $-0.43 \pm 0.04$ & $1.40 \pm 0.12$ & $-1.30 \pm 0.02$ & $1.09 \pm 0.16$ \\
\hline UGC 7408 & IAm & 6.70 & $-0.76 \pm 0.12$ & $-0.92 \pm 0.09$ & $-0.11 \pm 0.12$ & $0.66 \pm 0.02$ & $-1.17 \pm 0.18$ & $1.33 \pm 0.23$ \\
\hline
\end{tabular}

Note. ${ }^{\text {a }}$ Hubble types are from the NASA/IPAC Extragalactic Database (http://ned.ipac.caltech.edu); brackets indicate our revised classifications based on the high resolution images. Distances are from D. Calzetti et al. (2014, in preparation) assuming a Hubble constant of $70 \mathrm{~km} \mathrm{~s}^{-1} \mathrm{Mpc}^{-1}$.

high pressures influence cluster boundedness for all masses and at all levels in the hierarchy is unknown.

A related question is whether there is an upper cutoff in the cluster mass function. A cutoff of $\sim 10^{5}-10^{6} M_{\odot}$ was suggested for some spiral galaxies by Gieles et al. (2006), Bastian (2008), and Larsen (2009). Does the starburst NGC 1705 mentioned above have a normal cluster mass function but a higher mass cutoff, allowing a structure with $10^{6} M_{\odot}$ to become gravitationally bound? Do starburst galaxies in general have higher cutoffs, or no cutoffs as suggested for the Antennae galaxies by Whitmore et al. (2010)?

The formation of super star clusters (SSCs) in dwarf galaxies like NGC 1705 is also important to understand because metalpoor globular clusters probably formed in dwarf-like galaxies in the early universe (Chies-Santos et al. 2011; Elmegreen et al. 2012; Leaman et al. 2013). Such a formation site is suggested from the mass-metallicity relation of galaxies as a function of redshift (Mannucci et al. 2009). Perhaps SSCs in small galaxies reach high pressures during dwarf-dwarf galaxy mergers (Bekki 2008), or because of the ram pressure from accreting gas streams, as appears to be the case in NGC 1569 (Johnson et al. 2012) and NGC 5253 (López-Sánchez et al. 2012). These perturbations would be large-scale sources of turbulence, as opposed to stellar feedback, which is a smallscale source. The scale for turbulent energy injection may be evident from kinks or turn-overs in the scaling functions for turbulent motions and their resulting structures (Padoan et al. 2009).

These questions about the origin and boundedness of stellar groupings, cluster mass limits, and energy sources for high pressures and turbulence can be addressed with galaxies selected from the LEGUS survey (D. Calzetti et al. 2014, in preparation). Here we investigate multi-scale structure of star formation in 12 galaxies. We have shown previously that the distribution function of region size in a hierarchically structured region is a power law with a slope that is consistent with an interstellar medium partitioned by Kolomogorov-like turbulence and viewed in projection through a galaxy disk (Elmegreen et al. 2006). The purpose of this study is to see if the distribution functions for size and luminosity differ for starburst and normal systems.

Sánchez \& Alfaro (2008) studied the fractal dimension of H II region positions in dwarf irregular and spiral galaxies, finding that the brightest $\mathrm{H}$ II regions in any one galaxy have smaller fractal dimensions than the faintest $\mathrm{H}$ II regions (i.e., the brighter ones are more clumped together), and that in general the $\mathrm{H}$ II region population in brighter galaxies has a slightly smaller fractal dimension than it does in fainter galaxies (more clumpy). They did not consider starbursts, however.

Parodi \& Binggeli (2003) and Odekon (2006) determined the correlation dimension $d N(r) / d r$ for cumulative number of emission points $N$ as a function of distance $r$ from the centers of star-forming regions in dwarf Irregular galaxies. They found that the dimension increases for the brighter dwarfs-meaning that star formation is more area-filling, less strongly sub-clustered, and less porous for the brighter dwarfs. A similar variation of the power spectrum slope for $\mathrm{H} \alpha$ was found by Willett et al. (2005) in dwarf galaxies, where the power spectrum slope ranged from the Kolmogorov value characteristic of turbulence to shallower values as the filling factor of the $\mathrm{H} \alpha$ decreased. Evidently there is a characteristic power-law structure inside all of these star forming regions, and a dilution of this structure in the whole galaxy depending on the star formation filling factor.

We find a similar result here, that individual star-forming regions have steep number-versus-size relations in the NUV, and that the starburst dwarfs have similarly steep relations throughout their disks because of a dominance of these structures. The results of this study are in Section 3 and possible implications are in Section 4.

\section{OBSERVATIONS}

The Legacy Extragalactic UV Survey (LEGUS) is a Hubble Space Telescope (HST) Cycle 21 imaging survey in NUV, $U, B$, $V$, and $I$ of 50 nearby galaxies with WFC3/UVIS (D. Calzetti et al. 2014, in preparation). The survey is designed to include galaxies spanning different Hubble types. The pipeline data reduction is described by D. Calzetti et al. (2014, in preparation). In this study, we select 12 galaxies observed at F336W and $\mathrm{F} 275 \mathrm{~W}$ in order to examine the distribution of hierarchical structure in the youngest stars. Composite color images (F275W, $\mathrm{F} 336 \mathrm{~W}$, and $\mathrm{F} 435 \mathrm{~W}$ or $\mathrm{F} 438 \mathrm{~W}$ ) are shown for nine of the galaxies in Figure 1, while Hubble types and distances are in Table 1. Four of the galaxies are spirals and the rest are dwarf irregulars, with two having starburst characteristics and SSCs, NGC 1705 (Annibali et al. 2009) and NGC 5253 (Westmoquette et al. 2013). Other galaxies in LEGUS are not included because 

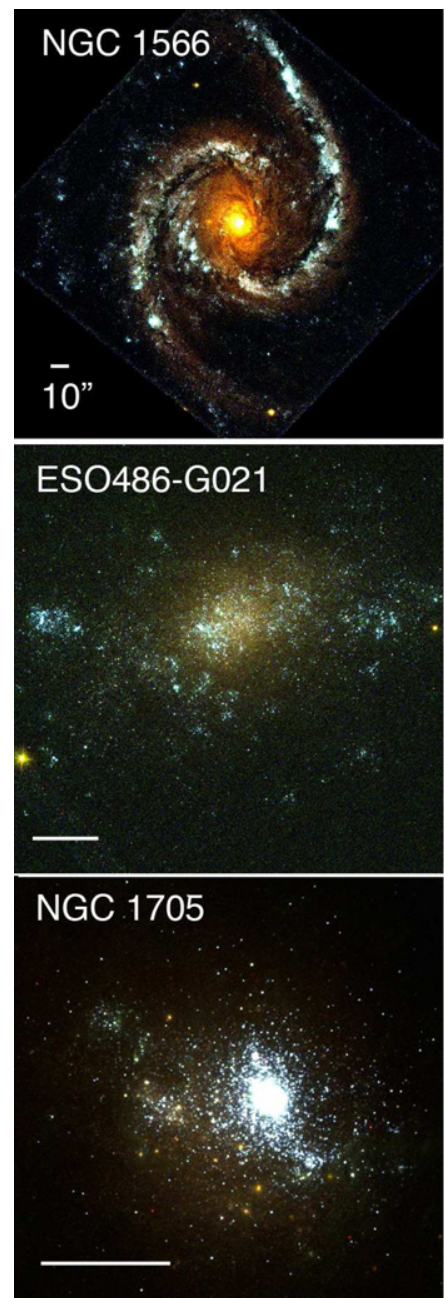
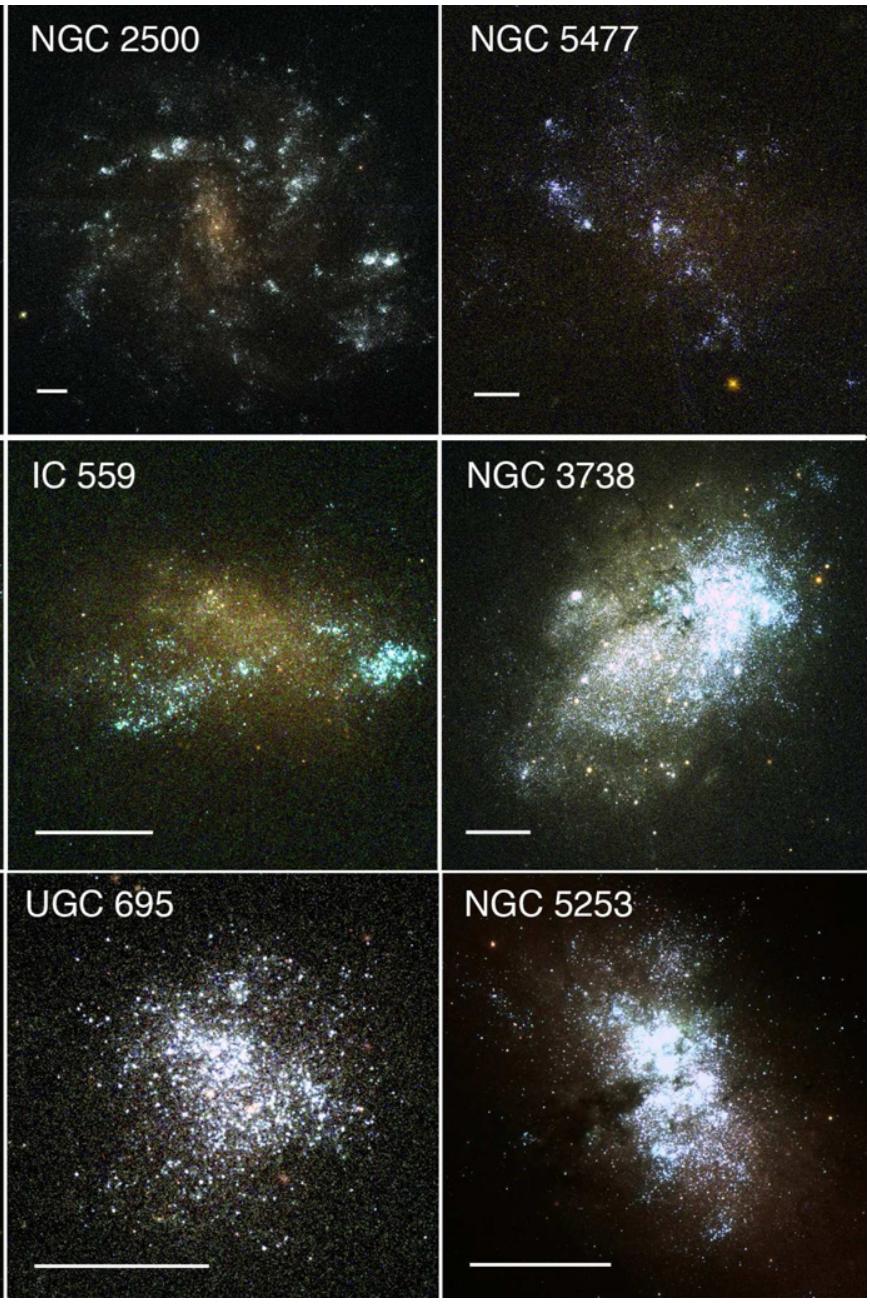

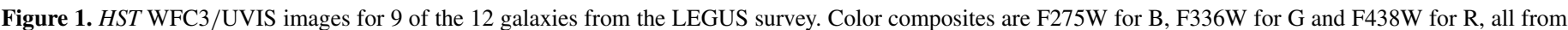
WFC3, except for NGC 5253 which uses F435W from the ACS. The scale bar is 10 arcsec.

(A color version of this figure is available in the online journal.)

they are either too highly inclined, incompletely sampled, or not observed yet.

\section{DATA ANALYSIS AND RESULTS}

In order to examine the number distribution function of star-forming regions as a function of size, the F336W and F275W images of each galaxy were smoothed with the Gaussian function gauss in IRAF using $\sigma$ values of 2, 4, 8, 16, 32, 64, and 128 pixels (the average FWHM of stars was measured to be $1.88 \pm 0.11$ pixels on the $\mathrm{F} 336 \mathrm{~W}$ image). A sample result is shown in Figure 2. The source extraction program SExtractor (Bertin \& Arnouts 1996) was used to make catalogs and source images from each blurred image. Different detection thresholds and minimum area thresholds were tried until realistic-looking source images were obtained. The fits used a minimum area of 10 pixels, a detection threshold of $10 \sigma$, a local background mesh 64 pixels wide, and a background filter 3 pixels wide.

The top panels of Figure 3 show the number of sources with a size greater than the abscissa values versus these sizes for structures in the F336W (left) and F275W (right) images. The slope in these plots is the projected fractal dimension of the star formation structure. The two filters give essentially the same results so there are no strong age effects. The starburst galaxies tend to have steeper slopes than the spiral and non- burst dwarfs, which means that the starbursts are more areafilling with lots of small regions inside and around the large regions. Recall that the dimension approaches the value of 2 as the projected image becomes totally covered. The slopes do not differ significantly between the spirals, which are dusty, and the non-starburst dwarfs, which have less dust, suggesting that extinction is not significant. Neither do the slopes differ because of the presence of spiral arms, because the largest scales considered here $(\sim 200 \mathrm{pc})$ are only comparable to the arm thicknesses and not to the arms' elongated shapes.

Linear least-squares fits to the correlations discussed here are listed in Table 1. The number-size relation just discussed is fitted by the expression $\log N=A_{\mathrm{NS}}+B_{\mathrm{NS}} \log S$ with slope coefficient $B$ in the table and subscript "NS" meaning "number-size." Others have a similar notation. In all cases, the fits are based on the smallest five scales where the correlations are most like power laws.

The middle left panel in Figure 3 shows the total flux of all the SExtractor-selected regions as a function of size. What is plotted on the ordinate is

$$
\log _{10} F=-0.4 M_{\mathrm{AB}}=\log _{10} C-0.4 \times 24.5377+2 \log _{10}\left(10^{5} D\right)
$$

for absolute specific flux $F$, absolute magnitude $M_{\mathrm{AB}}$, counts $C$, zero-point 24.5377 in the case of $F 336 \mathrm{~W}$, and distance $D$ in 


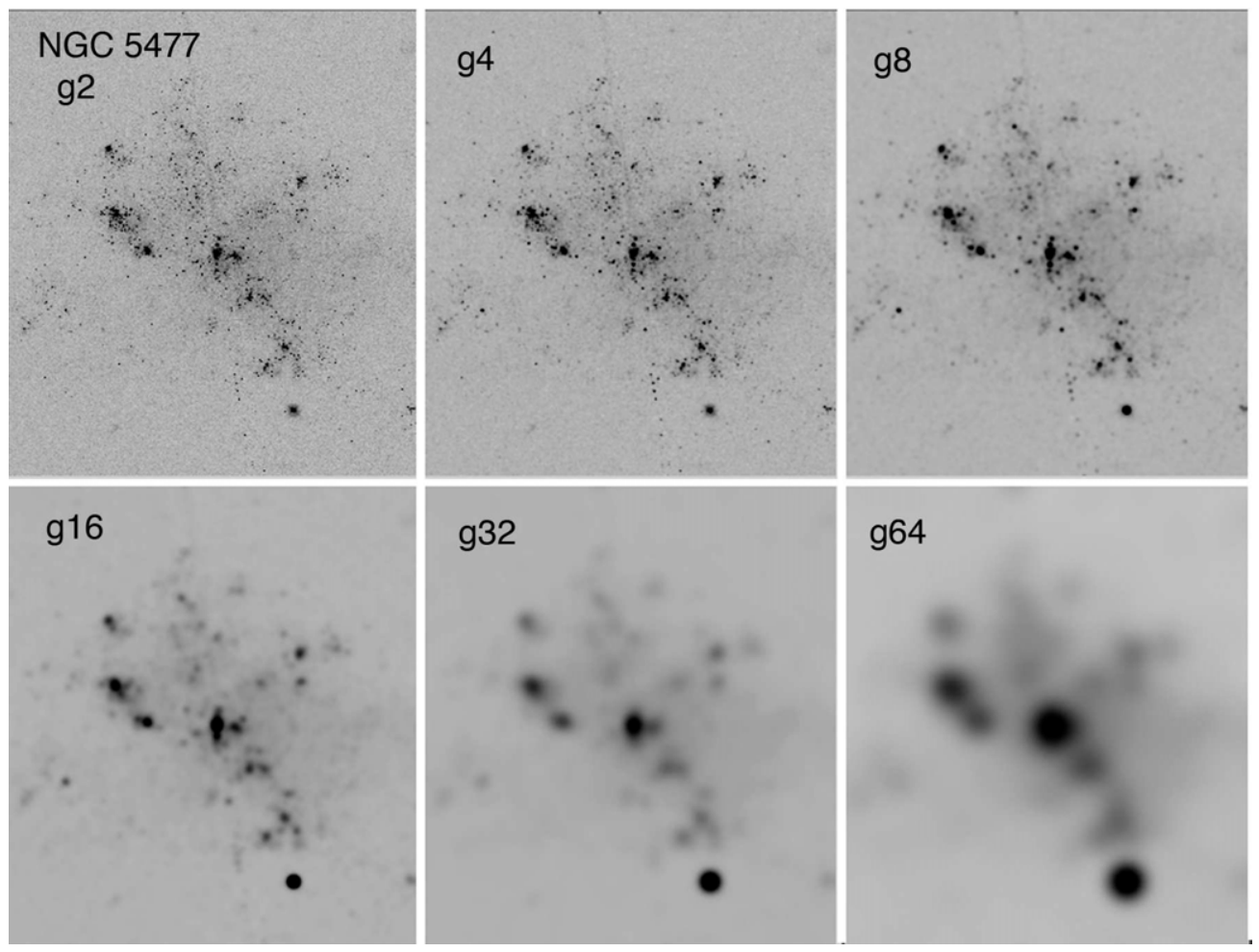

Figure 2. Smoothed images of NGC 5477 with Gaussian blurs of 2 pixels, 4, 8, 16, 32, 64 .

Mpc. The figure shows that the total flux decreases slightly with increasing size (slope $B_{\mathrm{TS}}$ in Table 1), which means that most of the selected regions are contained in one identified structure or another on all scales, except for the small and faint regions, which drop out successively as the blur size gets larger. The ratio of this total flux to the number of sources is the average flux per source; this is shown in the middle-right panel as a function of size (slope $B_{\mathrm{FS}}$ ). The starbursts again differ from the non-bursting galaxies because they have a steeper slope in average flux versus size. This corresponds to our impression that the starbursts have brighter regions on large scales than non-starburst galaxies. This brightening occurs systematically for all scales and not just suddenly at the largest. Because the total flux is nearly invariant with size for small sizes $\left(B_{\mathrm{TS}} \sim 0\right)$, the average flux-size correlation is approximately the inverse of the number-size correlation $\left(B_{\mathrm{FS}}=B_{\mathrm{TS}}-B_{\mathrm{NS}} \sim-B_{\mathrm{NS}}\right)$.

The lower left panel shows the flux distribution function ("the luminosity function," slope $B_{\mathrm{NF}}$ ), which replots the ordinate of the number-size relation versus the ordinate of the average-flux-size relation (the twist at the bottom of each plot is from the drop in the total flux at large scales, which is from the loss of faint and small features). If all of the flux in these structures were present at all scales, then the total flux would be constant with size, $B_{\mathrm{TS}}=0$, and the slope of the flux distribution function would be $B_{\mathrm{NS}} / B_{\mathrm{FS}}=B_{\mathrm{NS}} /\left(B_{\mathrm{TS}}-B_{\mathrm{NS}}\right)=-1$ (for $\log$ intervals) independent of the fractal dimension (which is $-B_{\mathrm{NS}}$ ). Not all of the flux is present on all scales however $\left(B_{\mathrm{TS}}<0\right)$, because the smaller and fainter sources that are outliers of the bigger and brighter sources drop below the $10 \sigma$ threshold for inclusion as the Gaussian blur size increases. NGC 1705 has a flux distribution function slope that is shallower by $4 \sigma$ compared to the others, and also a maximum flux that is nearly an order of magnitude larger than for the others, reflecting the presence of the SSC.
To assess how much of the star formation lies outside of the hierarchy, masks were made on one scale, e.g., the 32 pixel blur, and then the regions on a factor-of-two smaller scale that are inside and outside the masks were determined. Figure 4 shows the inner and outer regions of size 16 pixels (i.e., compared to the 32 pixel mask) for NGC 5477.

The lower right panel of Figure 3 shows the outlier fraction more systematically, plotting the luminosity fractions of regions on a scale of $N$ pixels that are outside the regions having a scale of $N+1$ pixels, versus the scale of $N$ pixels. All of the galaxies have an increasing outlier fraction with size (slope $B_{\mathrm{OS}}$ in Table 1) except for NGC 1705 and UGC 695, which have similarly rising fractions for small size and then a drop to zero fraction (the drop begins at the large dot in the figure). Such a drop indicates a concentration of essentially all of the bright star formation in one large region, as is also evident from Figure 1.

Power law slopes for the number-size relation were determined in nine subregions of seven galaxies out to typically 8 or 16 pixel blurs, depending on the region size. The galaxies were NGC 2500, IC 4247, NGC 5253, NGC 5477 (two regions), NGC 7793, NGC 3738, and IC 559 (two regions). The slopes were steep for all subregions, averaging $B_{\mathrm{NS}}=1.97 \pm 0.29$.

\section{DISCUSSION AND CONCLUSIONS}

The UV images in this survey show two distinct morphologies. One is characteristic of the large spiral galaxies and low surface brightness dwarfs where there is patchy and distributed star formation and low emission between the patches. There is no obvious hierarchical structure among the different patches, which seem to be independent or strung out along spiral arms, but there is hierarchical structure inside of them, to the extent that it can be resolved (e.g., NGC 7793). The other morphology is characteristic of starburst dwarfs or H II galaxies where 

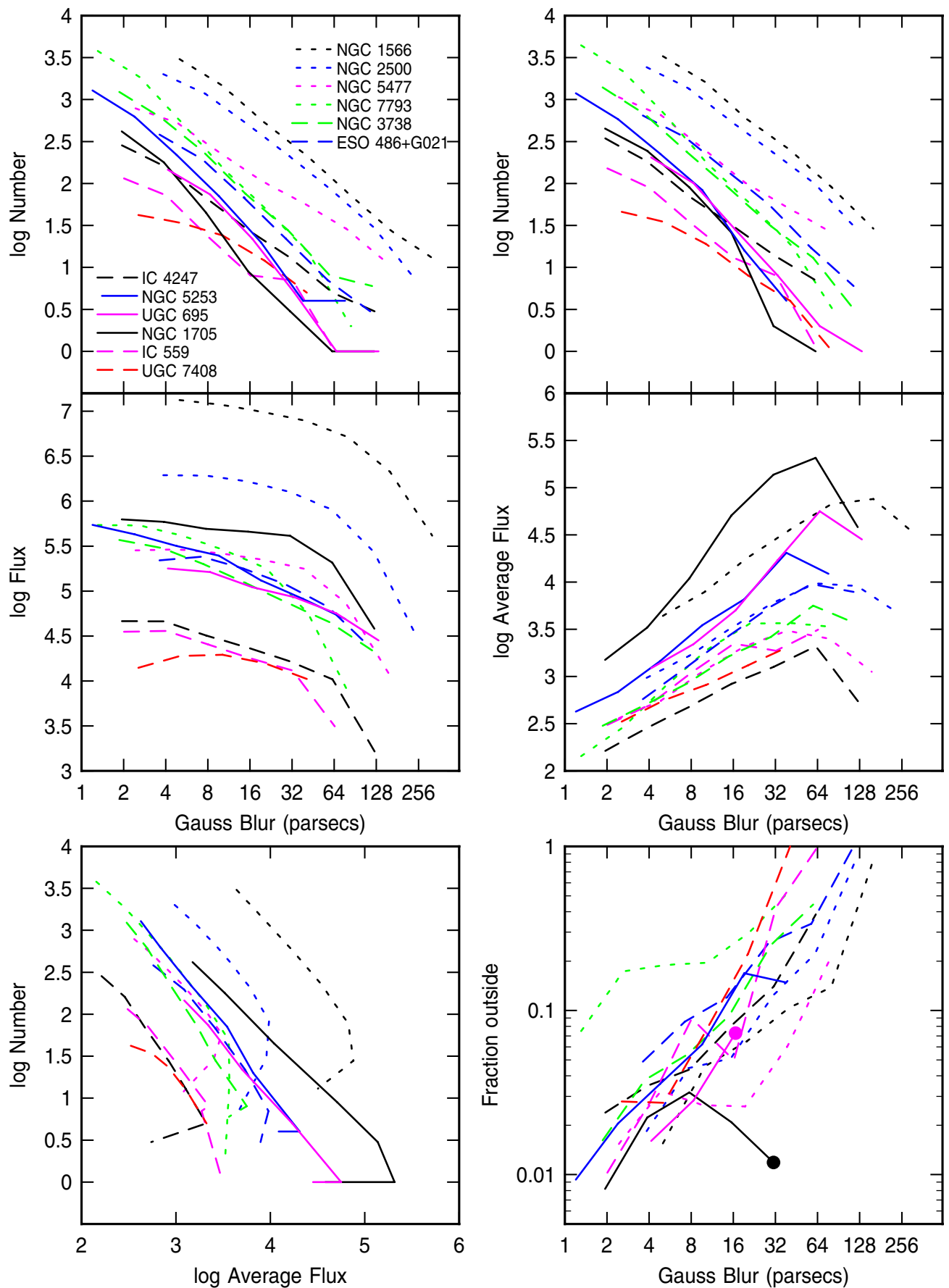

Figure 3. Size and flux distribution functions for star-forming regions found by SExtractor. Top left: the number of regions larger than size $S$ (in parsecs) vs. $S$, from the F336W images. The galaxies corresponding to each line type are indicated; line types are roughly divided into spirals (dotted), dwarfs (dashed) and starbursts (lines). Top right: cumulative number vs. size from the F275W images. Middle left: total flux at F336W in all SExtractor-selected regions larger than $S$ vs. $S$. Middle right: the ratio of the total flux at F336W to the number of regions larger than $S$ vs. $S$; this is the average F336W flux per region. Bottom left: the number of regions vs. their average F336W flux. Bottom right: the fraction of the F336W flux in SExtractor-selected regions on the plotted scale $S$ that are outside of the SExtractor-selected regions on the next-larger scale, $2 S$.

(A color version of this figure is available in the online journal.)

the image is dominated by one or two patches of star formation, which seem large relative to the size of the galaxy. These patches are well resolved and clearly hierarchical inside. We identify these ultraviolet patches with giant star complexes such as those studied by Efremov (1995).

The hierarchical structure observed by the number-size distribution or the flux-size distribution is approximately scale free up to the largest scale, as shown by the good power- law fits. The corresponding fractal dimension is large for the individual complexes too, which means a steep number-size slope approaching the limit of two for a completely filled and nested region. The fractal dimension is almost this large for the whole galaxies that are dominated by one or two complexes (NGC 1705, NGC 5253, UGC 695). Galaxies of the first morphological type have small fractal dimensions (shallow slopes). 


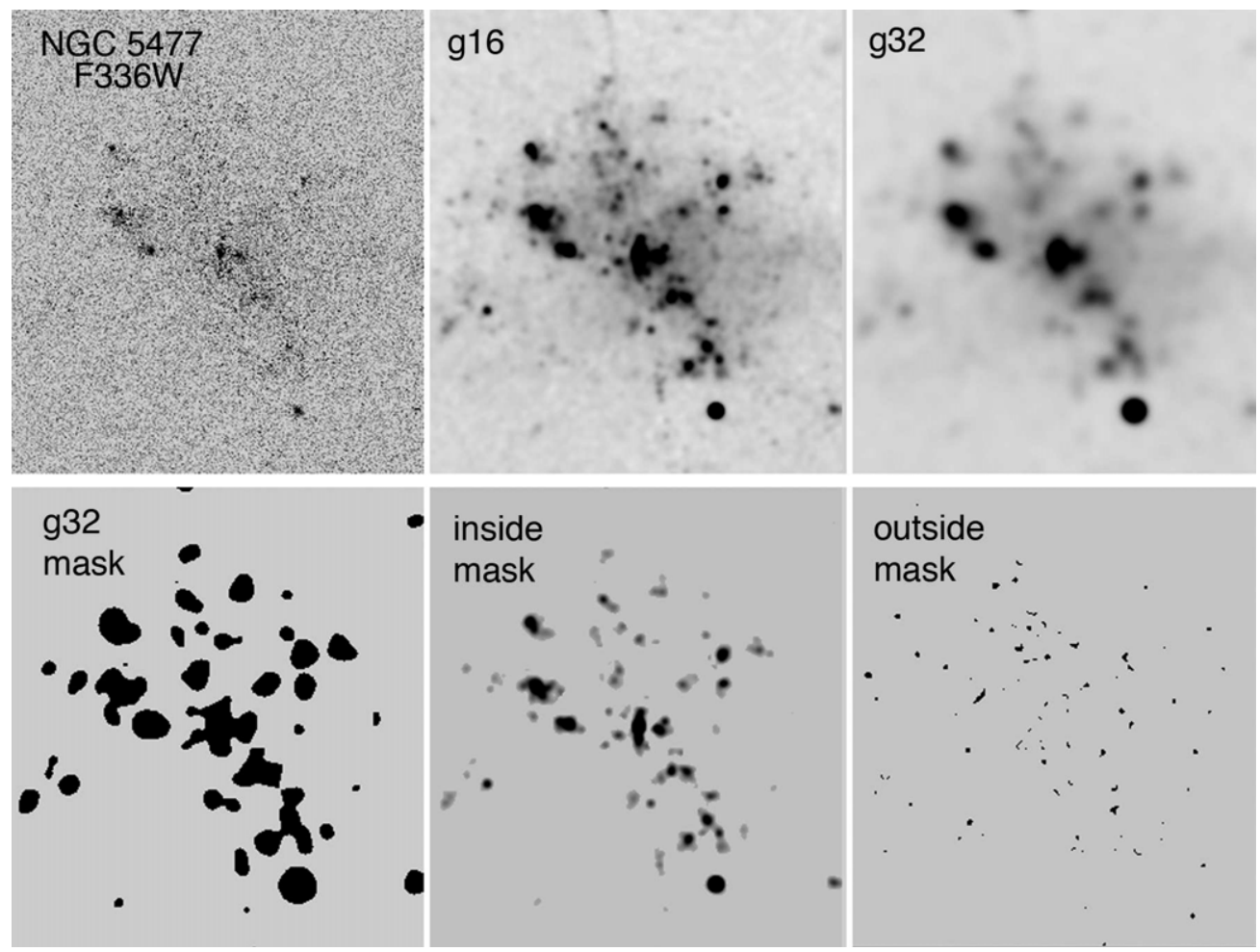

Figure 4. NGC 5477 with Gauss blurs of 16 and 32 pixels (left to right, top), the mask made from the 32 pixel blurred image (lower left), and the g16 sources inside and outside the mask boundaries.

The galaxies dominated by single large complexes also tend to have most of their smaller regions inside their larger regions, which means that the fractional luminosity from outliers goes to zero on large scales. In the other galaxies, this fraction monotonically increases with scale because the complexes are spread out and get lost with increased blurring as isolated regions (outliers) rather than as embedded regions.

The power-law structure of star-forming regions in these galaxies is consistent with the standard model where star formation is regulated by turbulent processes, such as gas compressions that form successively smaller clouds inside and around larger clouds ("turbulent fragmentation"; VázquezSemadeni et al. 2009). Such processes form a similar hierarchy of young stars, with a likely secondary correlation for star age, making larger regions older in proportion to the turbulent crossing time (Efremov \& Elmegreen 1998; de la Fuente Marcos $\&$ de la Fuente Marcos 2009a). The hierarchy has an upper limit in size beyond which separate regions form independently. This is consistent with the observation that the two-point correlation for stars and clusters decreases as a power law with increasing scale up to about one kpc (Scheepmaker et al. 2009; Bastian et al. 2011).

The starbursts in our sample also have SSCs, especially NGC 1705 and NGC 5253. A high projected density of hierarchical star formation should play a role in the formation of these clusters because smaller stellar groupings more readily coagulate and attract each other in a crowded environment, especially in low-mass galaxies where the binding energy in the star-forming cloud is a large fraction of the gravitational potential in the disk at that location. Moreover, because these structures are power laws, such coagulation should happen all throughout the cluster mass range, preserving the cluster mass distribution function. It should affect primarily the largest cluster mass that can form, which should increase in such a region.

Minniti et al. (2004) suggest a coagulation origin for an SSC in NGC 5128. This interpretation is also consistent with the finding by Annibali et al. (2009) that the stars $10-15 \mathrm{Myr}$ old in NGC 1705 are closer to the (coeval) SSC than the younger stars $(<5 \mathrm{Myr})$, and that there are many other smaller clusters nearby. In galaxies with more dispersed star formation, the only remnants of this hierarchical process could be cluster pairs (Dieball et al. 2002; de la Fuente Marcos \& de la Fuente Marcos 2009b).

A shift in the correlated properties of young stars around the star-forming region NGC 346 in the Small Magellanic Cloud, from one that is fractal on large scales to one that is centrally concentrated with a power-law density profile in the core region, suggests an analogous change in gas density structure when selfgravity becomes important in a turbulent medium (Gouliermis et al. 2014).

In conclusion, star formation observed in ultraviolet images with HST shows hierarchical structure from scales of a few hundred parsecs down to the parsec scale of individual bound clusters. The clusters therefore appear to form in the densest parts of a self-gravitating cloud complex that is structured by turbulence. Starburst dwarfs tend to have most of their ultraviolet structure in this form because they have one or two dominant young star complexes that are each hierarchical inside. Spiral galaxies and low surface brightness dwarfs have more uniformly dispersed complexes. The presence of dense hierarchical structure in a galaxy-dominant star complex would seem to favor an increase in the largest mass cluster that can form without changing the power-law slope of the mass function for the lower mass clusters. This may be the origin of the Schechtertype mass function that has been observed for clusters, and it 
may also explain the apparent variations in the cutoff mass as a function of environment.

Based on observations made with the NASA/ESA Hubble Space Telescope, obtained at the Space Telescope Science Institute, which is operated by the Association of Universities for Research in Astronomy, Inc., under NASA contract NAS 5-26555. These observations are associated with program No. 13364 (LEGUS), including grants HST-GO-13364.15-A (D.M.E.) and HST-GO-13364.14-A (B.G.E.). This research has made use of the NASA/IPAC Extragalactic Database (NED) which is operated by the Jet Propulsion Laboratory, California Institution of Technology under contract with NASA. D.A.G. kindly acknowledges financial support by the German Research Foundation through grant GO 1659/3-1.

\section{REFERENCES}

Adamo, A., Östlin, G., \& Zackrisson, E. 2011, MNRAS, 417, 1904

Annibali, F., Tosi, M., Monelli, M., et al. 2009, AJ, 138, 169

Bastian, N. 2008, MNRAS, 390, 759

Bastian, N., Weisz, D. R., Skillman, E. D., et al. 2011, MNRAS, 412, 1539

Bekki, K. 2008, MNRAS, 388, L10

Bertin, E., \& Arnouts, S. 1996, A\&AS, 117, 393

Chies-Santos, A. L., Larsen, S. S., Kuntschner, H., et al. 2011, A\&A, 525, A20

de la Fuente Marcos, R., \& de la Fuente Marcos, C. 2009a, ApJ, 700, 436

de la Fuente Marcos, R., \& de la Fuente Marcos, C. 2009b, A\&A, 500, L13

Dieball, A., Müller, H., \& Grebel, E. K. 2002, A\&A, 391, 547

Efremov, Y. N. 1995, AJ, 110, 2757

Efremov, Y. N., \& Elmegreen, B. G. 1998, MNRAS, 299, 588

Elias, F., Alfaro, E. J., \& Cabrera-Cao, J. 2009, MNRAS, 397, 2

Elmegreen, B. G. 2008, ApJ, 672, 1006

Elmegreen, B. G. 2010, in IAU Symp. 266, Star Clusters Basic Galactic Building Blocks Throughout Time And Space, ed. R. de Grijs \& J. Lepine (Cambridge: Cambridge Univ. Press), 3

Elmegreen, B. G., Elmegreen, D. M., Chandar, R., Whitmore, B., \& Regan, M. 2006, ApJ, 644, 879
Elmegreen, B. G., Elmegreen, D. M., \& Leitner, S. 2003, ApJ, 590, 271

Elmegreen, B. G., Malhotra, S., \& Rhoads, J. 2012, ApJ, 757, 9

Feitzinger, J. V., \& Braunsfurth, E. 1984, A\&A, 139, 104

Gieles, M., Larsen, S. S., Scheepmaker, R. A., et al. 2006, A\&A, 446, L9

Goddard, Q. E., Bastian, N., \& Kennicutt, R. C. 2010, MNRAS, 405, 857

Gomez, M., Hartmann, L., Kenyon, S. J., \& Hewett, R. 1993, AJ, 105,1927

Gouliermis, D. A. 2011, PhyS, 84, 048401

Gouliermis, D. A., Hony, S., \& Klessen, R. S. 2014, MNRAS, 439, 3775

Hunter, D. A., O'Connell, R. W., Gallagher, J. S., \& Smecker-Hane, T. A. 2000, AJ, 120, 2383

Johnson, M., Hunter, D. A., Oh, S.-H., et al. 2012, AJ, 144, 152

Kennicutt, R. C., Jr. 1998, ApJ, 498, 541

Kritsuk, A. G., Lee, C. T., \& Norman, M. L. 2013, MNRAS, 436, 3247

Larsen, S. 2009, A\&A, 494, 539

Larsen, S. S., \& Richtler, T. 2000, A\&A, 354, 836

Larson, R. B. 1995, MNRAS, 272, 213

Leaman, R., VandenBerg, D. A., \& Mendel, J. T. 2013, MNRAS, 436, 122

López-Sánchez, Á. R., Koribalski, B. S., van Eymeren, J., et al. 2012, MNRAS, 419, 1051

Maíz-Apellániz, J. 2001, ApJ, 563, 151

Mannucci, F., Cresci, G., Maiolino, R., et al. 2009, MNRAS, 398, 1915

Minniti, D., Rejkuba, M., Funes, J. G., \& Kennicutt, R. C., Jr. 2004, ApJ, 612,215

Nguyen Luong, Q., Motte, F., Schuller, F., et al. 2011, A\&A, 529, A41

Odekon, M. C. 2006, AJ, 132, 1834

Padoan, P., Juvela, M., Kritsuk, A., \& Norman, M. L. 2009, ApJL, 707, L153

Parmentier, G., \& Fritze, U. 2009, ApJ, 690, 1112

Parodi, B. R., \& Binggeli, B. 2003, A\&A, 398, 501

Sánchez, N., \& Alfaro, E. J. 2008, ApJS, 178, 1

Scheepmaker, R. A., Lamers, H. J. G. L. M., Anders, P., \& Larsen, S. S 2009, A\&A, 494, 81

Tan, J. C., Shaske, S. N., \& Van Loo, S. 2013, in IAU Symp. 292, Molecular Clouds: Internal Properties, Turbulence, Star Formation and Feedback (Cambridge: Cambridge Univ. Press), 19

Vázquez-Semadeni, E., Gómez, G. C., Jappsen, A.-K., Ballesteros-Paredes, J., \& Klessen, R. S. 2009, ApJ, 707, 1023

Westmoquette, M. S., James, B., Monreal-Ibero, A., \& Walsh, J. R. 2013, A\&A, 550, A88

Whitmore, B. C., Chandar, R., Schweizer, F., et al. 2010, AJ, 140, 75

Willett, K. W., Elmegreen, B. G., \& Hunter, D. A. 2005, AJ, 129, 2186 